\title{
MILP-based short-term thermal unit commitment and hydrothermal scheduling including cascaded reservoirs and fuel constraints
}

\author{
Mohamed Shaaban ${ }^{1}$, Hossein Zeynal' ${ }^{2}$, Khalid Nor ${ }^{3}$ \\ ${ }^{1}$ Department of Electrical \& Electronic Engineering, Faculty of Engineering, Universiti Malaysia Sarawak, Malaysia \\ ${ }^{2}$ Faculty of Mathematics \& Computer Science, Buein Zahra Technical University, Iran \\ ${ }^{3} \mathrm{KMN}$ Consultants, Malaysia
}

\begin{tabular}{l}
\hline \hline Article Info \\
\hline Article history: \\
Received May 3, 2018 \\
Revised Dec 17, 2018 \\
Accepted Mar 11, 2019 \\
\hline
\end{tabular}

\section{Keywords:}

Hydrothermal power generation Integer-linear programming Multithreading

Optimal scheduling

Power grids

\begin{abstract}
Reservoirs are often built in cascade on the same river system, introducing inexorable constraints. It is therefore strategically important to scheme out an efficient commitment of thermal generation units along with the scheduling of hydro generation units for better operational efficiency, considering practical system conditions. This paper develops a comprehensive, unit-wise hydraulic model with reservoir and river system constraints, as well as gas constraints, with head effects, to commit thermal generation units and schedule hydro ones in the short-term. A mixed integer linear programming (MILP) methodology, using the branch and bound \& cut $(\mathrm{BB} \& \mathrm{C})$ algorithm, is employed to solve the resultant problem. Due to the detailed modelling of individual hydro units and cascaded dependent reservoirs, the problem size is substantially swollen. Multithread computing is invoked to accelerate the solution process. Simulation results, conducted on various test systems, reiterate that the developed MILP-based hydrothermal scheduling approach outperforms other techniques in terms of cost efficiency.
\end{abstract}

Copyright $(2019$ Institute of Advanced Engineering and Science. All rights reserved.

\section{Corresponding Author:}

Mohamed Shaaban,

Department of Electrical and Electronic Engineering,

Faculty of Engineering, Universiti Malaysia Sarawak,

Kota Samarahan 94300, Sarawak, Malaysia.

Email: mshaaban@unimas.my

\section{INTRODUCTION}

Day-to-day operations of a utility requires efficient load forecasting to carry out unit commitment, economic dispatch, hydrothermal coordination, and load management [1]. Hydro-thermal scheduling (HTS) plays a pivotal role in power system operation. HTS optimally dispatches thermal and hydro generation plants throughout the scheduling horizon, in the most economical way, subject to overall operational constraints and practical system limits [2]. Economic considerations such as fuel utilization and total operation cost of the system can be substantially influenced by the proper allocation of water resources in multiple river systems. This is particularly imperative in countries with limited hydropower resources.

HTS is basically a large-scale, nonlinear mixed integer programming problem with complicating constraints [2], [3]. The presence of detailed hydraulic modeling of the river and reservoirs coupled with the non-convexity of the HTS problem, makes it complex owing to the increased number of binary variables and coupling constraints. As the problem becomes more intricate for practical systems of large sizes, it is extremely difficult to come up with consistent optimal schedules. The latter may not only be computationally onerous, but also insurmountable in some cases.

Various solution techniques were proposed in the literature. While AI-based approaches have received increased attention [4], utilities and independent system operators (ISOs) widely use Dynamic Programming 
(DP) [5], Lagrangian Relaxation (LR) [3, 6] and Mixed-Integer Linear Programming (MILP) methods [7-13].

DP is a stage-wise search for an optimal solution, upon dividing the main problem into smaller subproblems. The optimal solution of the original problem involves making a sequence of optimal decisions on the subproblems [14]. Nevertheless, as the dimension of the problem may increase substantially with the problem size, DP could become numerically unstable [5]. Lagrangian Relaxation (LR) also decomposes the original problem into subproblems; one per thermal unit and one subproblem per hydro plant. LR then evaluates the dual problem through the decomposed primal problem, incorporating Lagrange multipliers of global variables, by solving the subproblems of thermal units and hydroelectric ones [3]. Since individual thermal and hydro units are modeled as separate subproblems in LR, devising the coupling constraints can be formidable. As compared with DP, LR does not suffer from the curse of dimensionality, albeit encountering instances of unnecessary commitment of units; rendering higher production costs [15]. Additionally, heuristic reasoning is required to meet the duality gap [6].

Mixed-integer linear programming (MILP), using Branch and Bound and Cut (BB\&C) algorithm, is a powerful tool to solve combinatorial optimization problems [16]. Since MILP obviates the need for heuristic reasoning and provides a direct mathematical modeling of the coupling constraints, it surpasses DP and LR approaches [15]. MILP converts the associated nonlinearities of the HTS model, through a piecewise linearization, into a set of linear inequalities. CPLEX, an off-the-shelf software for solving large-scale MILP problems using BB\&C algorithm, can then be applied [8, 10, 11, 13, 15].

In many of the HTS investigations, hydro units in a plant were commonly aggregated as one unit [7]. While this reduces the total number of decision variables, resulting in a less computationally demanding problem, it may, on the other hand, impair the accuracy of the solution. In an actual hydro plant, and at a certain instant during the scheduling period, some units may be committed, while others are shut down. In addition, in some cases, cascaded reservoirs over the same river basin were assumed to be independent, such that the complexity of the model becomes more manageable [3, 17].

This paper averts the above issues and proposes a comprehensive, fine-grained system model. An MILP-based approach for thermal unit commitment and scheduling of hydro generation units is developed, using the $\mathrm{BB} \& \mathrm{C}$ algorithm. The contribution of this paper is in three major aspects. First, an elaborate hydraulic model for the river and cascaded reservoirs system with complicating constraints (unit-wise, head effect, cascaded reservoirs, riparian, fuel) is developed. Second, an MILP formulation for the presented hydro-thermal scheduling (HTS) model is carried out. Non-convex production cost, start-up/shut-down cost, and the hydro performance curve, are all linearized accurately producing a finer cut and a narrower search space. Last, to make the HTS solution more efficient and make the maximal use of existing resources, multithreading is exploited to enhance computational performance of the solution. This can only be achieved due to the way the problem is structured, to make it amenable for parallel processing. Consequently, very large systems, with thorough hydraulic modeling, can be solved in a relatively short time.

The organization of the paper is as follows: Section 2 presents the formulation of the HTS problem. Section 3 briefly describes the linearization of the production cost, stair-wise start-up cost and shut-down cost models of the MILP-HTS problem. Case studies and numerical results are demonstrated in Section 4. Section 5 provides conclusions.

\section{HYDROTHERMAL SCHEDULING PROBLEM}

The HTS problem can be mathematically formulated as:

\subsection{Objective function}

The objective of the HTS scheduling is to minimize the production cost, start-up cost, and shut-down costs of all generating units, over the scheduling horizon $T$. The objective function is constrained by demand and reserve requirements [2].

$$
\operatorname{Min} . \sum_{t \in T} \sum_{j \in J} c_{j}^{p}(t)+s_{j}^{s u}(t)+c_{j}^{s d}(t), \quad \forall t \in T, \forall j \in J
$$

Subject to

$$
\sum_{j \in J} p_{j}(t)+\sum_{i \in I} p_{i}(t)=D(t) \forall t \in T, \forall j \in J, \forall i \in I
$$




$$
\begin{aligned}
& \sum_{j \in J} y(t) p_{j}^{\max }(t)+\sum_{i \in I} \gamma(t) p_{i}^{\max }(t) \geq D(t)+S R(t) \\
& p_{j}(t) \in \Delta_{j}(t), \quad \forall t \in T, \forall j \in J \\
& p_{i}(t) \in \Delta_{i}(t), \quad \forall t \in T, \forall i \in I
\end{aligned}
$$

In the above formulation, production cost includes maintenance costs as a fixed component per unit energy. The operation cost of hydro units is not accounted for in the above formulation, since it is negligible.

The cost function is subject to the power balance equations, (2) and (3), such that, total generation from hydro and thermal units must match demand and spinning reserve (10\% of the load at this paper) at each interval. The model also encompasses instant reserves that can be available in five minutes only. The latter can be expressed in the same way as spinning reserves (3). The demand $D(t)$ includes transmission losses as well. Initially, losses are accounted for as fixed, but get updated in subsequent iterations. The feasible region that each thermal and hydro unit is permitted to operate within are represented by (4) and (5). Unit-wise limitations such as ramp up/down, minimum up/down, and unit's generation capacity fall under these equations.

\subsection{Thermal constraints}

$$
\begin{aligned}
& P_{j}^{\min } y_{j}(t) \leq p_{j}(t) \leq P_{j}^{\max } y_{j}(t), \forall t \in T, \forall j \in J \\
& p_{j}(t)-p_{j}(t-1) \leq R U_{j}, \forall t \in T, \forall j \in J \\
& p_{j}(t-1)-p_{j}(t) \leq R D_{j}, \forall t \in T, \forall j \in J \\
& \sum_{t \in T} \sum_{j \in J} V_{j}^{g}(t) \leq V_{t o t a l}, \quad \forall t \in T, \forall j \in J \\
& V_{j}^{g}=a_{j} y_{j}(t)+b_{j} p_{j}(t)+c_{j} p_{j}^{2}(t)
\end{aligned}
$$

Equation (6) expresses the upper/lower bounds for thermal generation unit output, whereas the capability of each unit to ramp up/down can be modeled as (7)-(8). A thermal unit $j$ can ramp up/down from the current/preceding interval to the consecutive/current time-interval less than a pre-specified rate of ramping $R U_{j} / R D_{j}$. Minimum up/down time constraints and spinning reserve allocation are detailed in [8].

One of the coupling constraints, that couple all thermal generators in the mix, is the limited fuel availability over the scheduling horizon. The total use of gas over a time span, $T$, is less than the available gas volume (9). The fuel consumption is represented by a quadratic function (10).

\subsection{Hydraulic constraints}

The two kinds of hydraulic constraints, fixed-head hydro unit and variable head hydro unit, are considered to demonstrate the hydraulic head variation effect. The fixed-head hydro unit can be expressed as [17]:

$$
\begin{aligned}
& p_{i}(t) \propto f\left(q_{i}(t)\right), \quad \forall t \in T, \forall i \in I \\
& p_{i}(t)=a_{i} \gamma_{i}(t)+b_{i} q_{i}(t)+c_{i} q_{i}^{2}(t)
\end{aligned}
$$

Hydropower generation depends on the amount of water discharged from hydro units, (11)-(12). For the case of large reservoir capacity, in practice and for short-term scheduling, hydraulic head variation can be ignored, and assumed to be constant.

The variable-head hydro unit can be represented as [18]:

$$
p_{i}(t) \propto f\left(q_{i}(t), h_{i}(t)\right), \quad \forall t \in T, \forall i \in I
$$


Hydro generation levels change dynamically with hydraulic head variations, which, in turn affect the model considered [15]. Such variations are due to the difference between the natural inflow and water discharge. More specifically, the hydropower generated from a generating unit is a function of the water discharge variation as well as the water content (head level) of the associated reservoir [18]. These relationships are expressed mathematically in (11)-(13).

In this paper, the model in [18] is extended to become a unit-based hydrothermal scheduling problem as opposed to earlier models, which used to be merely a plant-based approach. The implementation of hydro unit-based head variation effect is far more challenging, where a certain number of hydro units are associated with a specific reservoir. This would consequently increase the number of constraints on the one hand, but on the other hand, would enable a precise representation of actual reservoir performance in river systems.

\subsection{Water volume constraints}

$$
\begin{aligned}
& \sum_{t=1}^{T} q_{i}(t) \leq Q_{i}, \forall t \in T, \forall i \in J \\
& \sum_{t \in T} \sum_{i \in I} q_{i}(t) \leq Q_{\text {total }}, \quad \forall t \in T, \forall i \in I
\end{aligned}
$$

The above constraints ensure that the water released from individual units, and all units, follow the hydro operator plan-a practical consideration usually associated with operation of hydro power plants.

\subsection{Hydraulic cascade operation}

In many countries around the world, the operation of hydro generation units can be optimized by extending the model to include the operation of hydraulically-coupled reservoirs over a river catchment. Such constraint establishes a codependency relationship between upstream and downstream hydro units that enables the better use of scarce hydro resources.

$$
\begin{aligned}
& X_{i}(t)=X_{i}(t-1)+\eta_{i}(t)+\sum_{m=\ddot{Y}}\left[q_{m}\left(t-\tau_{m i}\right)+s_{m}\left(t-\tau_{m i}\right)\right] \\
& -q_{i}(t)-s_{i}(t), \quad \forall t \in T, \forall i \in I, i \in \ddot{Y}
\end{aligned}
$$

The water continuity at each reservoir in the cascaded mode operation of a river system follows a linear relationship as illustrated in (16). The water content of reservoir $X$ associated with hydro unit $i$ is depleted by the water discharged from the same reservoir $i$, and spillage water $s_{i}(t)$. On the other hand, the content of the reservoir is dynamically filled by the natural inflow $\eta_{i}(t)$, and the water released from upstream units associated with the reservoir. However, the amount of water reaches the reservoir in the downstream side, after a delay time $\tau_{m i}$, is $q_{m}\left(\mathrm{t}-\tau_{m i}\right)$. Such codependency between upstream and downstream reservoirs introduces significant challenges to the HTS solution process.

\subsection{Reservoir content limit}

The maximum and minimum capacities of the reservoir to store water are $\bar{X}_{i}$ and $\underline{X}_{i}$ respectively. This can be expressed mathematically as:

$$
\underline{X}_{i} \leq X_{i}(t) \leq \bar{X}_{i}, \quad \forall t \in T, \forall i \in I
$$

\subsection{Discharge limits}

To avoid any possible mechanical damage to the hydro turbine, the water released from each hydro unit should be maintained within an upper bound and a lower bound $\bar{q}_{i}$ and $\underline{q}_{i}$ respectively. This can be expressed as:

$$
\underline{q}_{i} \gamma_{i}(t) \leq q_{i}(t) \leq \bar{q}_{i} \gamma_{i}(t), \quad \forall t \in T, \forall i \in I
$$

Whereas the minimum discharge of a hydro unit is theoretically zero, delimiting the discharge to zero is inefficient and may increase the wear and tear of windings and mechanical equipment. In addition, it could lead to control device malfunction [18]; incurring a higher maintenance cost. In good utility practices, the water discharge from each hydro generation unit usually exceeds a pre-specified minimum limit. 


\subsection{Riparian constraint}

The water flowing through the channel connecting two consecutive reservoirs on a river system has to be controlled. The aim of this constraint is to curb possible damages that may occur to facilities and communities alongside the river stream. In addition, to provide a minimum flow for navigational and irrigational purposes (if needed), while maintaining the water outflow of hydro plants within a specified range.

$$
\underline{q}_{i} \gamma_{i}(t) \leq \sum_{i}^{n} q_{i}(t) \leq \bar{q}_{i} \gamma_{i}(t), \quad \forall t \in T, \forall i \in I
$$

Equation (19) is referred to as riparian constraint, which represents an important practical restriction for utilities to be held. Instead of controlling the water flow in the channel, the outflow of each hydro plant, containing several units, can be restricted.

To summarize, (1)-(19) represent an inclusive HTS problem with a meticulous hydraulic modeling. This optimization problem is unequivocally high dimensional, particularly with utility-sized systems, that requires a long processing time. In fact, the resulting mixed-integer nonlinear programming problem, (1)-(19) is insolvable, particularly for a weekly scheduling problem with half an hour intervals. These models are very difficult to solve by conventional nonlinear programming methods. Hence, a mixed-integer linear programming (MILP) formulation, is proposed for the HTS model, using branch, bound and cut (BB\&C) algorithm. Additionally, multithreading which can accelerate the solution process with concurrent threads, is also described.

\section{MILP-HTS FORMULATION}

The solution of the above HTS formulation is formidable as it involves many nonlinear characteristics in the objective function as well as the constraints. The nonlinear components are the quadratic production cost, hydro performance curve, exponential start-up cost, shut-down cost, and fuel availability. These nonlinear constraints can be linearized to form an equivalent MILP formulation, so that it can be solved efficiently.

To cater for the nonlinearity retained by the mixed-integer nonlinear model, a piecewise approximation is employed to transform all nonlinear functions and constraints to an equivalent linearized model. Consequently, large-scale problems can be moderated and managed efficiently.

\subsection{Linearized production cost}

Equation (20) of the quadratic production cost (\$-MW) can be piecewise linearly approximated, (21)-(26), by contemplating sufficient number of breakpoints [8]. A single type of binary variable $y_{j}(t)$ to model the entire MILP formulation is deemed sufficient [8]. Choosing an adequate number of breakpoints in the horizontal axis makes the piecewise linear representation almost identical to the original nonlinear curve, as demonstrated in Figure 1. On the one hand, increasing the number of breakpoints for piecewise linearization can definetly improve the approximation accuracy. On the other hand, with increased number of break points, the problem size may be swollen and the computational process could be compromised. Four piecewise segments provide adequate modeling of the quadratic cost function, rendering a solution with acceptable accuracy and in a moderate time [8], [15]. In the above formulation, variable operating cost; namely the maintenance cost of the unit that increases when the amount of generation ramps up, is incorporated into (21).

With the same argument articulated for (20)-(26), the quadratic non-concave power vs discharge (p-q) of the hydro performance curve, (11)-(13), and the fuel consumption quadratic function, (10), can be similarly approximated to a set of linear equations, if adequate piecewise segments were used [18].

$$
\begin{gathered}
c_{j}^{p}(t)=a_{j} y_{j}(t)+b_{j} p_{j}(t)+c_{j} p_{j}^{2}(t), \forall t \in T, \forall j \in J \\
c_{j}^{p}(t)=A_{j} y_{j}(t)+\sum_{l=1}^{N l_{j}} F_{l j} \mu_{l}(j, t), \forall t \in T, \forall j \in J \\
p_{j}(t)=P_{j}^{\min } y_{j}(t)+\sum_{l=1}^{N l_{j}} \mu_{l}(j, t), \forall t \in T, \forall j \in J
\end{gathered}
$$




$$
\begin{aligned}
& \mu_{1}(j, t) \leq T_{1 j}-P_{j}^{\mathrm{min}}, \forall t \in T, \forall j \in J \\
& \mu_{l}(j, t) \leq T_{l j}-T_{l-1 j}, \forall t \in T, \forall j \in J, \forall l=2 \ldots N l_{j-1} \\
& \mu_{N l_{j}}(j, t) \leq P_{j}^{\max }-T_{N l_{j-1}}, \forall t \in T, \forall j \in J \\
& \mu_{l}(j, t) \geq 0, \forall t \in T, \forall j \in J, \forall l=1 \ldots N l_{j} \\
& A_{j}=a_{j}+b_{j} P_{j}^{\min }+c_{j}\left(P_{j}^{\min }\right)^{2}
\end{aligned}
$$

\subsection{Linearized start-up and shut-down costs}

A typical exponential startup cost function is shown by the dashed- line curve in Figure 2. The startup cost has become a discrete function, due to the discretization of the time span into hourly periods (the black dots). The discrete start-up cost can be approximated by a stair-wise function as described in (27)-(28). The linearization mechanism for stair-wise start-up cost and step-wise shut-down costs, which depends only on the binary variables, corresponds to the on/off state of generating units, (27)-(30). The total number of binary variables of the proposed MILP-HTS will be much lesser, since it does not contain separate binary variables assigned for start-up and shut-down states. This will reduce the problem size as well as the processing time [5].

$$
\begin{aligned}
& c_{j}^{s u}(k) \geq K_{j}^{t}\left[y_{j}(k)-\sum_{n=1}^{t} y_{j}(k-n)\right] a_{j} y_{j} \\
& \forall j \in J, \forall k \in K, \forall t \in T, \forall t=1 \ldots N D_{j} \\
& c_{j}^{s u}(k) \geq 0, \forall t \in T, \forall j \in J \\
& c_{j}^{s u}(k) \geq C_{j}\left[y_{j}(k-1)+y_{j}(k)\right], \forall j \in J, \forall k \in K \\
& c_{j}^{s d}(k) \geq 0, \forall j \in J, \forall k \in K
\end{aligned}
$$

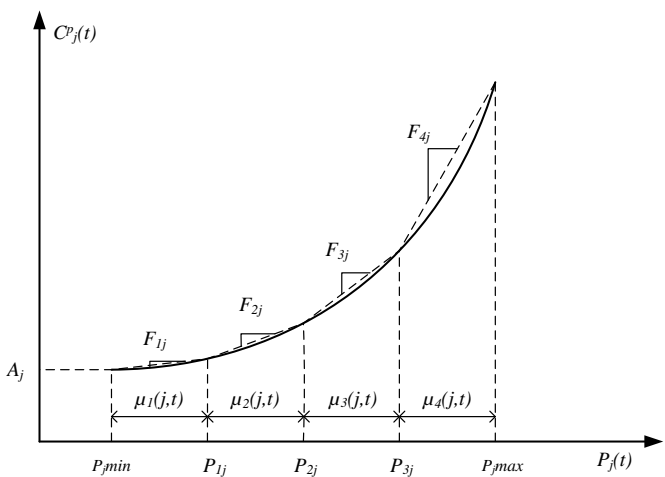

Figure 1. Cost model piecewise approximation

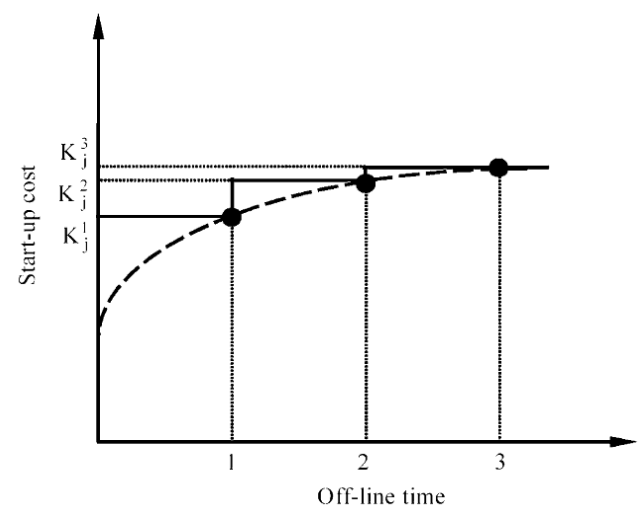

Figure 2. Stair-wise start-up cost functions

\section{NUMERICAL RESULTS}

Several systems are tested to demonstrate the efficacy and superiority of the proposed methodology relative to other techniques from the literature. Simulation was implemented through CPLEX® 12.4, employing the branch, bound and cut $(\mathrm{BB} \& \mathrm{C})$ method, running on Intel coreTM i5 processor with $2.67 \mathrm{GHz}$ speed and 6 GB of RAM. The developed model is first compared against other major approaches. Comparisons are conducted over the same test system comprising 100 thermal units [5]. This system, however, is a purely thermal unit commitment system. 
Three hydrothermal test systems are designated to test the proposed MILP-HTS algorithm. The test systems are: IEEE 300-bus with 69 units [19], a practical utility system of 777-bus that contains 132 units, and a modified ERCOT (Energy Reliability Council of Texas) system with 4995 bus comprising 287 units. Details of each test system case study components and characteristics are listed in Table 1. In the first case, hydro plants are built on a single river catchment as shown in Figure 3, representing hydraulic coupling of plants over a waterway.

Table 1. Hydrothermal test systems

\begin{tabular}{lccc}
\hline \multicolumn{1}{c}{ Instance } & Case I & Case II & Case III \\
\hline Number of buses & 300 & 777 & 4995 \\
Number of units & 69 & 132 & 287 \\
Number of thermal units & 51 & 96 & 233 \\
Number of hydro units & 18 & 36 & 54 \\
Number of Reservoirs & 5 & 10 & 15 \\
Number of river networks & 1 & 2 & 3 \\
\hline
\end{tabular}

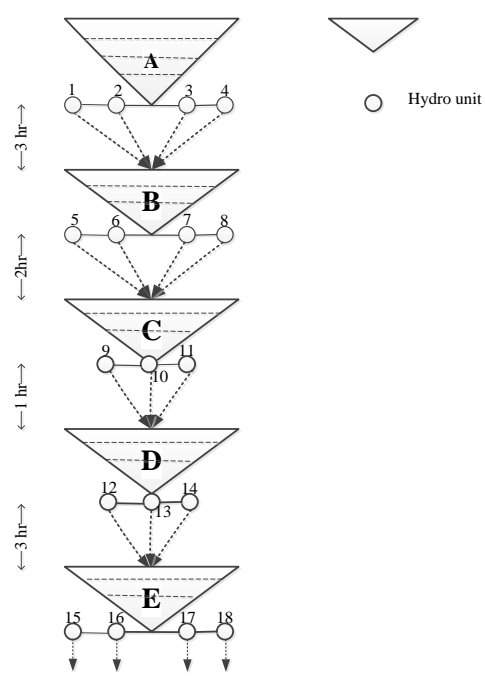

Figure 3. River system topology in waterway connection

For the second case with 132 units, hydro plants data of the 69-unit case are replicated to make two distinctive cascaded hydro chains ( 2 river networks with 36 units). Similarly, the hydro network, in case III with 287-unit, is made up by replicating case I hydro network for three times. As a result, the 287-unit contains 3 cascaded chains of hydro units, in which every chain has 18 hydro generation units.

\subsection{Comparison with other algorithms}

To validate the performance of the proposed MILP methodology, a comparison against other techniques, reported in the literature, is carried out. For this case, a pure thermal unit commitment problem, without hydro units, is solved for a 100-unit benchmark system. Table 2 illustrates the performance of several techniques; namely the Genetic Algorithm (GA) [20], Particle Swarm Optimization (PSO) [21], Lagrangian Relaxation (LR) [22], straightforward algorithm (SF) [23], and Extended Priority Listing (EPL) [24]. It is conspicuous, from Table 2, that the proposed MILP-based technique offers an exceptional solution that outshines all other approaches, with the cheapest cost of $\$ 5,594,011$.

Table 2. Comparison between Different Algorithms

\begin{tabular}{lcc}
\hline Technique & Total operation cost [\$] & Cost difference [\%] \\
\hline GA & $5,627,437$ & 0.60 \\
PSO & $5,625,376$ & 0.56 \\
LR & $5,620,305$ & 0.47 \\
SF & $5,615,960$ & 0.39 \\
EPL & $5,608,440$ & 0.26 \\
Proposed MILP & $5,594,011$ & 0.00 \\
\hline
\end{tabular}




\subsection{Head variation effect}

Simulation results, for the three test systems portrayed in Tables 3 and 4, are based on a typical daily load data. An optimality gap of $0.5 \%$ was presumed in all test cases. An optimality gap between $0-1 \%$ is always recommended as a valid industrial choice [15]. From the results demonstrated in Table 3 and 4 , it is evident that when the reservoir head changes were modeled (i.e., variable head model) the operation cost of each system has decreased noticeably. The operation cost of the 69-unit has diminished from $\$ 2,401,951$ to $\$ 2,398,425$, marking a $\$ 3526$ reduction in total cost for a daily load, based on an hourly interval. The trend is reiterated in the other two large systems as well. These results clearly assert the consequence of the detailed variable head modeling and its direct return on daily cost savings. Nonetheless, while accurate head modeling, as shown in Table 3 and 4, rationalizes the costs and makes better use of water content in reservoirs, it adds extra variables, resulting in higher computational burden $[25,26]$.

Table 3. Solution performance without hydraulic head variation

\begin{tabular}{lccc}
\multicolumn{4}{c}{ hydraulic head variation } \\
\hline \multirow{2}{*}{ Instance } & \multicolumn{3}{c}{ Fixed Head Hydro } \\
& Case I & Case II & Case III \\
\hline No. of variables & 15888 & 30480 & 64944 \\
No. of binary & 1656 & 3168 & 6888 \\
variables & & 29457 & 62960 \\
No. of constraints & 15387 & 2960 \\
No. of NNZ in & 54004 & 103064 & 222139 \\
constraint matrix & & & \\
Time (sec) & 5.610 & 54.880 & 415.270 \\
Cost (M\$) & 2.400 & 6.322 & 7.100 \\
\hline
\end{tabular}

Table 4. Solution performance with hydraulic head variation

\begin{tabular}{|c|c|c|c|}
\hline \multirow{2}{*}{ Instance } & \multicolumn{3}{|c|}{ Variable Head Hydro } \\
\hline & Case I & Case II & Case III \\
\hline No. of variables & 17184 & 33072 & 68832 \\
\hline $\begin{array}{l}\text { No. of binary } \\
\text { variables }\end{array}$ & 2520 & 4896 & 9480 \\
\hline No. of constraints & 18723 & 36129 & 72968 \\
\hline $\begin{array}{l}\text { No. of NNZ in } \\
\text { constraint matrix }\end{array}$ & 75172 & 143672 & 283051 \\
\hline Time (sec) & 238.050 & 447.730 & 679.670 \\
\hline Cost (M\$) & 2.390 & 6.312 & 7.088 \\
\hline
\end{tabular}

\subsection{Multithreading}

Multithread computing is a parallel processing technology used to reduce the computation time. It is carried by sharing process resources concurrently across multiple processors at different stages of execution [27]. Parallel processing was used in hydro power scheduling [28], and microgrid power management system [29]. In addition, multithreading was applied successfully in unit commitment [25] and variable wind generation scheduling [30].

Table 5 summarizes the executional performance for two of the case studies. In this case, the optimality gap is tightened to $0.1 \%$, instead of $0.5 \%$ in previous cases, and the computational performance of a 24-hour interval HTS is recorded. The serial or sequential execution is contrasted with the parallel execution via a number of threads. With a tighter gap, the execution time increases. Yet, it is clear from Table 5 that multithread computing can consistently improve the performance as compared with the serial execution. Doubling the number of threads, however, may not necessarily lead to significantly enhancing the computational time, albeit providing several orders of magnitude of solution speedup, paticularly in Case II.

The implementation of multithreading on the BB\&C process incurs splitting a number of branches in the search tree simultaneously. In some cases, the solution in a specific branch is dependent on the result from other branches. Therefore, the computing thread in the current branch has to remain idle, until the solution from other branches is obtained. This may affect the solution process to be sluggish in some instances. In Table 5, the fastest performance was found through 4-threads. Further research is needed to explore the multithreading application in the HTS problem.

Table 5. Multithread MILP-HTS computational performance

\begin{tabular}{cccc}
\hline \multirow{2}{*}{ \#Case } & \multicolumn{3}{c}{ Execution time (Sec) } \\
& Serial & \#Thread & \#Thread \\
& execution & 2 & 4 \\
\hline I & 116.10 & 54.92 & 38.46 \\
II & 309.64 & 303.0 & 40.15 \\
\hline
\end{tabular}

\section{CONCLUSIONS}

Hydrothermal scheduling is a formidable engineering problem that includes a plethora of complicating constraints. Due to such intricacy, hydro generation used to be cast aggregately, in a plant-wise framework to avoid complicating the problem further. However, in power systems with limited hydropower resources, it is imperative to model the hydro generation in a unit-wise manner to better reflect the practical system structure and the underlying operating conditions. A mixed-integer linear programming (MILP) 
methodology is presented in this paper to commit thermal and hydro generation units. A meticulous model of the hydraulic system including cascaded dependent reservoirs, variable reservoir head, riparian effects, individual hydro units, and fuel constraints is adopted. Since such extensive modeling could hamper the computational efficiency of the proposed methodology, multithread computing is utilized to enhance the computational capability. Application of the proposed methodology, using the branch and bound \& cut $(B B \& C)$ algorithm, on various test systems and under different conditions, has consistently manifested that the proposed methodology surpasses other techniques in terms of cost efficiency. It is also capable of solving largescale problems in a very competitive computation time.

\section{ACKNOWLEDGEMENTS}

This work was supported in part by the Malaysian Ministry of Higher Education (MOHE) and Universiti Malaysia Sarawak (UNIMAS), Malaysia under the Fundamental Research Grant Scheme (FRGS), Grant No. F02/FRGS/1620/2017.

\section{REFERENCES}

[1] I. A. Ethmane, M. Maaroufi, A. K. Mahmoud, and A. Yahfdhou, "Optimization for Electric Power Load Forecast," International Journal of Electrical and Computer Engineering (IJECE), vol/issue: 8(5), pp. 3453-3462, Oct. 2018.

[2] A. J. Wood, B. F. Wollenberg, and G. B. Sheble, Power Generation, Operation, and Control, 3rd ed.: Wiley, pp. 147$186,2013$.

[3] M. S. Salam, K. M. Nor, and A. R. Hamdam, "Hydrothermal Scheduling Based Lagrangian Relaxation Approach to Hydrothermal Coordination," IEEE Transactions on Power Systems, vol/issue: 13(1), pp. 226-235, Feb. 1998.

[4] S. S. Sakthi, R. K. Santhi, N. M. Krishnan, S. Ganesan, and S. Subramanian, "Wind Integrated Thermal Unit Commitment Solution using Grey Wolf Optimizer," International Journal of Electrical and Computer Engineering (IJECE), vol/issue: 7(5), pp. 2309-2320, Oct. 2017.

[5] R. W. Ferrero, J. F. Rivera, and S. M. Shahidehpour, "Dynamic Programming Two-Stage Algorithm for Long-Term Hydrothermal Scheduling of Multireservoir Systems," IEEE Transactions on Power Systems, vol/issue: 13(4), pp. 1534-1540, Nov. 1998.

[6] H. Zeynal, L. X. Hui, Y. Jiazhen, M. Eidiani, and B. Azzopardi, "Improving Lagrangian Relaxation Unit Commitment with Cuckoo Search Algorithm," in Proc. IEEE Int. Conf. Power and Energy (PECon), Kuching, Sarawak, Malaysia, pp. 77-82, 2014.

[7] G. W. Chang, M. Aganagic, J. G. Waight, J. Medina, T. Burton, S. Reeves, and M. Christoforidis, "Experiences with Mixed Integer Linear Programming Based Approaches on Short-Term Hydro Scheduling," IEEE Transactions on Power Systems, vol/issue: 16(4), pp. 743-749, Nov. 2001.

[8] M. Carrión and J. M. Arroyo, “A Computationally Efficient Mixed-Integer Linear Formulation for the Thermal Unit Commitment Problem,” IEEE Transactions on Power Systems, vol/issue: 21(3), pp. 1371-1378, Aug. 2006.

[9] J. Ostrowski, M. F. Anjos, and A. Vannelli, "Tight Mixed Integer Linear Programming Formulations for the Unit Commitment Problem," IEEE Transactions on Power Systems, vol/issue: 27(1), pp. 39-46, Feb. 2012.

[10] H. Zeynal and M. Eidiani, "Hydrothermal Scheduling Flexibility Enhancement with Pumped-Storage Units," in Proc. 22nd Iranian Conf. Electrical Engineering (ICEE), Tehran, Iran, pp. 820-825, 2014.

[11] W.-S. Tan, M. P. Abdullah, and M. Shaaban, "Chance-Constrained Scheduling of Variable Generation and Energy Storage in a Multi-Timescale Framework," Journal of Electrical Engineering \& Technology, vol/issue: 12(5), pp. 1709-1718, Sep. 2017.

[12] A. Moreira, G. Strbac, R. Moreno, A. Street, and I. Konstantelos, "A Five-Level MILP Model for Flexible Transmission Network Planning under Uncertainty: A Min-Max Regret Approach," IEEE Transactions on Power Systems, vol/issue: 33(1), pp. 486-501, Jan. 2018.

[13] M. Shaaban, W.-S. Tan, and M. P. Abdullah, "A Multi-Timescale Hybrid Stochastic/Deterministic Generation Scheduling Framework with Flexiramp and Cycliramp Costs," International Journal of Electrical Power \& Energy Systems, vol/issue: 99, pp. 585-593, July 2018.

[14] A. Lew and H. Mauch, Dynamic Programming: A Computational Tool, vol. 38: Springer, pp. 3-44, 2006.

[15] T. Li and M. Shahidehpour, "Price-Based Unit Commitment: A Case of Lagrangian Relaxation Versus Mixed Integer Programming," IEEE Transactions on Power Systems, vol/issue: 20(4), pp. 2015-2025, Nov. 2005.

[16] J. E. Mitchell, "Branch-and-Cut Algorithms for Combinatorial Optimization Problems," in Handbook of Applied Optimization, P. Pardalos and M. Resende, Eds., 1st ed., New York: Oxford University Press, pp. 65-77, 2002.

[17] S. Salam, K. M. Nor, and A. R. Hamdan, "Comprehensive Algorithm for Hydrothermal Coordination," IEE Generation, Transmission and Distribution, vol/issue: 144(5), pp. 482-488, Sep. 1997.

[18] A. J. Conejo, J. M. Arroyo, J. Contreras, and F. A. Villamor, "Self-Scheduling of a Hydro Producer in a Pool-Based Electricity Market," IEEE Transactions on Power Systems, vol/issue: 17(4), pp. 1265-1272, Nov. 2002.

[19] R. Christie. Power Systems Test Case Archive. 1993. [Online]: https://www2.ee.washington.edu/research/pstca/pf300/pg_tca300bus.htm. [Last Access] 12/11/2018.

[20] S. A. Kazarlis, A. G. Bakirtzis, and V. Petridis, "A Genetic Algorithm Solution to the Unit Commitment Problem," IEEE Transactions on Power Systems, vol/issue: 11(1), pp. 83-92, Feb. 1996. 
[21] B. Zhao, C. X. Guo, B. R. Bai, and Y. J. Cao, "An Improved Particle Swarm Optimization Algorithm for Unit Commitment," International Journal of Electrical Power \& Energy Systems, vol/issue: 28(7), pp. 482-490, Sep. 2006.

[22] H. H. Balci and J. F. Valenzuela, "Scheduling Electric Power Generators using Particle Swarm Optimization Combined with the Lagrangian Relaxation Method," International Journal of Applied Mathematics and Computer Science, vol/issue: 14(3), pp. 411-421, Mar. 2004. [Online] http://eudml.org/doc/207707. [Last Access] 12/11/2018.

[23] S. H. Hosseini, A. Khodaei, and F. Aminifar, "A Novel Straightforward Unit Commitment Method for Large-Scale Power Systems," IEEE Transactions on Power Systems, vol/issue: 22(4), pp. 2134-2143, Nov. 2007.

[24] T. Senjyu, K. Shimabukuro, K. Uezato, and T. Funabashi, "A Fast Technique for Unit Commitment Problem by Extended Priority List,” IEEE Transactions on Power Systems, vol/issue: 18(2), pp. 882-888, May 2003.

[25] W. S. Tan and M. Shaaban, "A Hybrid Stochastic/Deterministic Unit Commitment Based on Projected Disjunctive MILP Reformulation,” IEEE Transactions on Power Systems, vol/issue: 31(6), pp. 5200-5201, Nov. 2016.

[26] H. Zeynal, Implementation of Mixed Integer Linear Programming for Hydro-Thermal Generation Scheduling with River and Reservoir Constraints, PhD Thesis, Faculty of Electrical Engineering, Universiti Teknologi Malaysia, Malaysia, 2013. [Online]: http://eprints.utm.my/id/eprint/36661/. [Last Access] 12/11/2018.

[27] D. A. Zier and L. Ben, "Performance Evaluation of Dynamic Speculative Multithreading with the Cascadia Architecture," IEEE Transmission on Parallel and Distributed Systems, vol/issue: 21(1), pp. 47-59, Jan. 2010.

[28] G. Warland, A. L. Henden, and B. Mo, "Use of Parallel Processing in Applications for Hydro Power Scheduling Current Status and Future Challenges," Energy Procedia, vol/issue: 87, pp. 157-164, Jan. 2016.

[29] L. Dhanesh and P. Murugesan, "A Novel Approach in Scheduling of the Real-Time Tasks in Heterogeneous Multicore Processor with Fuzzy Logic Technique for Microgrid Power Management," International Journal of Power Electronics and Drive Systems (IJPEDS), vol/issue: 9(1), pp. 80-88, Mar. 2018.

[30] W. S. Tan, M. Shaaban, and M. P. Abdullah, "Chance-Constrained Programming for Day-Ahead Scheduling of Variable Wind Power Amongst Conventional Generation Mix and Energy Storage," IET Renewable Power Generation, vol/issue: 11(14), pp. 1785-1793, Dec. 2017.

\section{NOMENCLATURE}

\section{Variables}

$c_{j}^{p}(t) \quad$ : Production cost of thermal unit $j$ in period $t$.

$c_{j}^{s u}(t) \quad: \quad$ Start-up cost of thermal unit $j$ in period $t$.

$c_{j}^{s d}(t) \quad$ : Shut-down cost of thermal unit $j$ in period $t$.

$p_{j}(t) \quad: \quad$ MW output of thermal unit $j$ at period $t$.

$p_{i}(t) \quad: \quad$ MW output of hydro unit $i$ at period $t$.

$y_{j}(t) \quad: \quad$ Binary status (1/0) of thermal unit $j$ at period $t$.

$\mu_{l}(j, t) \quad$ : Power generation at block $l$ of piecewise linear function for unit $j$ at period $t$.

$V_{j}^{g}(t) \quad$ : Gas volume consumption by unit $j$ at period $t$.

$q_{i}(t) \quad: \quad$ Water discharge of hydro unit $i$ at period $t$.

$\gamma_{i}(t) \quad: \quad$ Binary status (1/0) for hydro unit $i$ at period $t$.

$X_{i}(t) \quad: \quad$ Reservoir associated to hydro unit $i$ at period $t$

$h_{i}(t) \quad: \quad$ Reservoir head for hydro unit $i$ at period $t$.

$q_{m}\left(t-\tau_{m i}\right):$ Discharge of hydro unit $i$ at period $t$ from upstream hydro units $m$ after traversal time $\tau$.

$s_{i}(t) \quad$ : Water spillage of the reservoir associated with hydro unit $i$ at period $t$.

\section{Constants}

$D(t)$

$S R(t)$

$\Delta_{j}(t)$

$\Delta_{i}(t)$

$a, b, c$

$\mathrm{A}$

$P_{j}^{\min }, P_{j}^{\max }$

$F_{l j}$
Demand at period $t$.

Spinning reserve amount at period $t$.

Feasible region for thermal unit $j$ at period $t$.

Feasible region for hydro unit $i$ at period $t$.

Coefficients of quadratic function.

Coefficient of piecewise linear function.

Min/Max generation of unit $j$.

Slope of segment $l$ in piecewise function for unit $j$. 


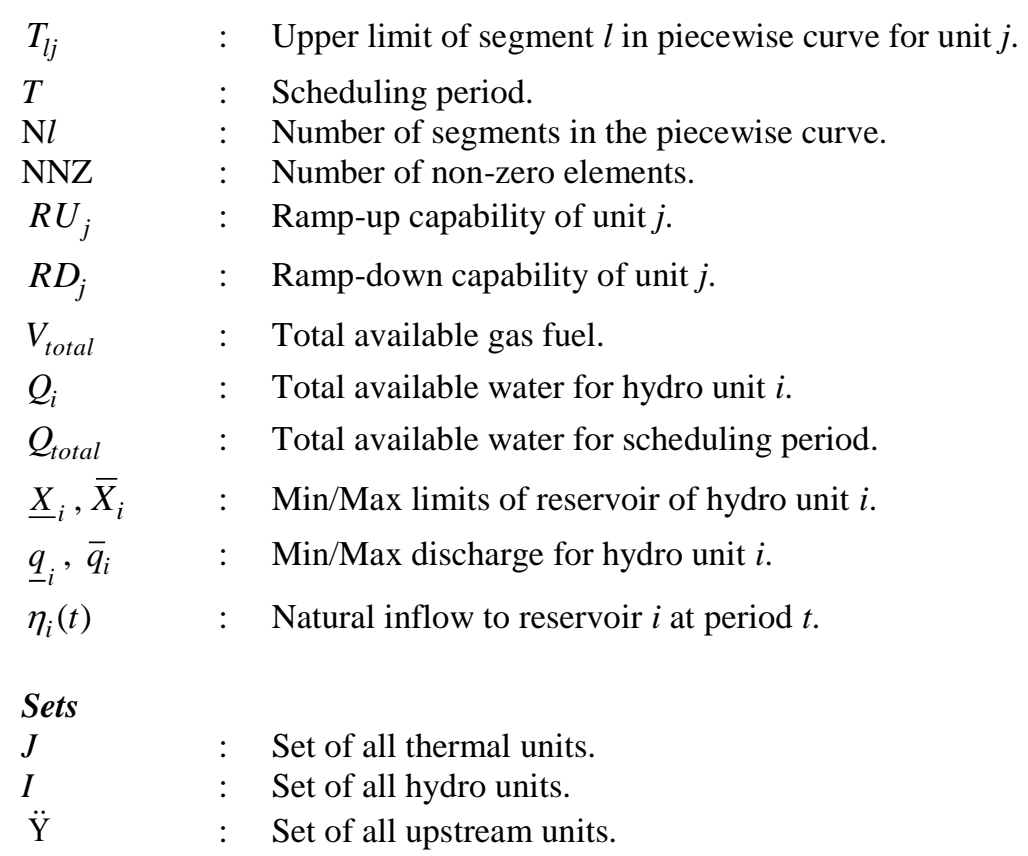

\section{BIOGRAPHIES OF AUTHORS}

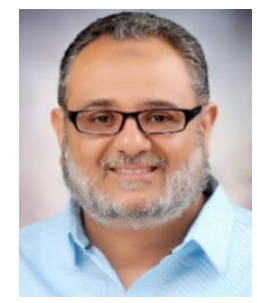

Mohamed Shaaban received his B.Sc. (honors) and M.Sc. degrees from Suez Canal University, Egypt and his Ph.D. degree (all in Electrical Engineering) from the University of Hong Kong in 1990, 1996, and 2002 respectively. He held previous appointments in Suez Canal University and Port Said University, Egypt, Qassim University, Saudi Arabia, University of Strathclyde, UK, and Universiti Teknologi Malaysia (UTM). He was a Visiting Professor at Washington State University, Pullman, WA, USA in 2007 and National Chung Cheng University, Taiwan in 2015. He is currently an Associate Professor in the Department of Electrical and Electronic Engineering, Universiti Malaysia Sarawak (UNIMAS). His current research interests include distributed generation scheduling, largescale optimization, microgrid operation, smart distribution grids, renewable energy integration, and big data analytics. Dr. Shaaban is a Chartered Engineer, UK and a Senior Member, IEEE.

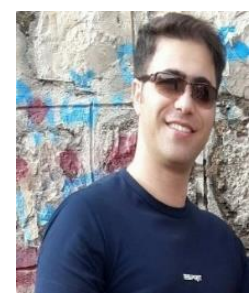

Hossein Zeynal was born in Bojnourd, Khorasan, Iran. He obtained his B.Eng. in Electrical Power Engineering from Azad University of Bojnourd, Iran in 2005, and his M.Eng. and Ph.D. degree from Universiti Teknologi Malaysia (UTM), Skudai, Johor, Malaysia in 2007 and 2013, both in Electrical Engineering (Power). He was a Lecturer at the School of Engineering, KDU University College, Damansara Jaya, Selangor, Malaysia. He is currently an Assistant Professor at the Deptartment of Electrical and Computer, Buein Zahra Technical University, Buein Zahra, Qazvin, Iran. His research interests include power system optimization, large-scale simulation, parallel computing, and Microgrid operation.

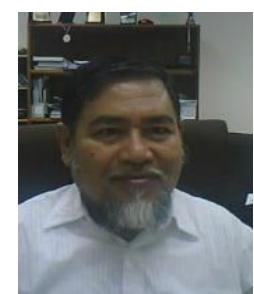

Khalid Nor received the B.Eng. degree (first-class honors) from the University of Liverpool, Liverpool, U.K., the M.Sc. degree in 1978, and the Ph.D. degree in 1981, both from the University of Manchester Institute of Science and Technology (UMIST), Manchester, U.K. He joined the University of Malaya, Kuala Lumpur, Malaysia, as a Lecturer in 1981. He was a Professor in the Department of Electrical Power Engineering, Universiti Teknologi Malaysia until he retired in 2012. He is currently an independent consultant. His research interests are in the field of electrical power system simulation, power quality, and unit commitment. Dr. Nor is a Senior Member, IEEE. 